\title{
Mortality salience effects fail to replicate in traditional and novel measures.
}

\author{
Bjørn Sætrevik \\ Operational psychology research group, Department for psychosocial science, Faculty of \\ Psychology, University of Bergen
}

\author{
Hallgeir Sjåstad \\ Department of Strategy and Management, Norwegian School of Economics, and SNF - Centre \\ for Applied Research at $\mathrm{NHH}$
}

\begin{abstract}
Mortality salience (MS) effects, where death reminders lead to ingroup-bias and defensive protection of one's worldview, have been claimed to be a fundamental human motivator. MS phenomena have ostensibly been identified in several hundred studies within the "terror management theory" framework, but transparent and high-powered replications are lacking. Experiment $1(\mathrm{~N}=101$ Norwegian lab participants) aimed to replicate the traditional MS effect on national patriotism, with additional novel measures of democratic values and pro-sociality. Experiment 2 ( $\mathrm{N}=784$ US online participants) aimed to replicate the MS effect on national patriotism in a larger sample, with ingroup identification and pro-sociality as additional outcome measures. The results showed that neither experiment replicated the traditional MS effect on national patriotism. The experiments also failed to support conceptual replications and underlying mechanisms on democratic values, processing speed, psychophysiological responses, ingroup identification, and pro-sociality. This indicates that the effect of death reminders is less robust and generalizable than previously assumed.
\end{abstract}

Keywords: Mortality salience, death reminders, worldview defence, terror management, replication.

The concept of mortality salience (MS) refers to a phenomenon where reminders of death lead to subconscious changes in attitudes and behaviour, typically in the form of increased ingroup-bias and behaviour that may serve the role of defending one's cultural worldview. The MS effect has been reported in several hundred experiments since the 1980's, with unusually large effect sizes (for meta-analyses, see: Burke et al., 2013; Burke et al., 2010). The dominant theoretical framework to account for MS effects has been the "terror management theory" (TMT, Greenberg et al., 1986;
Pyszczynski et al., 2015). This theory emerges from a psychodynamic approach to existential questions, and proposes automatic defence mechanisms that may protect the person from conscious death awareness. Specifically, TMT states that cognitions related to mortality evoke an aversive state of existential anxiety which motivates to suppress thoughts of vulnerability (proximal defences), or to bolster self-esteem or affirm cultural values to find meaning beyond death (distal defences). This has some similarities to the concept of "psychological defence mechanisms" in psychodynamic theory. It is 
mainly the MS effects of distal defence, often referred to as "cultural worldview defence", that has been investigated in social psychology experiments. In the terminology of TMT and the writings of Ernest Becker (1973), the general idea is that adhering to a cultural worldview can work as a buffer against the fear of death by providing a form of "symbolic immortality". The aim of the current study was to provide a high-powered and preregistered replication of the MS effect using traditional outcome measures, and to use novel outcome measures to examine possible mechanisms of MS.

Need for mortality salience replication. There is an ostensibly solid empirical basis for MS effects in terror management research, with a meta-analytical effect size of $d=0.82$ (Burke et al., 2010). However, the research tradition has also been called into doubt due to claimed theoretical weakness and non-falsifiability (Fiedler et al., 2012; Martin and Bos, 2014), researcher effects (Yen and Cheng, 2013), failure to replicate past findings (Trafimow and Hughes, 2012), and contradictory empirical findings (Hart, 2014). Given the recent method reform in psychological research and other fields (Munafò et al., 2017), it should also be noted that there have been few, if any, preregistered direct replications with open datasets showing robust MS effects.

As with other psychodynamic theories, the postulation of complex subconscious processes makes it challenging to empirically test MS effects. Past studies have used a variety of subtle experimental manipulations without appropriate manipulation checks. A review (Burke et al., 2010) is sometimes cited to argue that the MS effect is thoroughly empirically established. However, the review also reveals a great deal of variation in the experiment designs, in terms of different manipulations and different outcome measures, whether or not there are "delay tasks" (and their duration and number), and whether the MS effect relies on various covariates. Few of the studies report performing manipulation checks or provide open data. Despite the vast number of studies, no standard experiment approach for producing the MS effect appears to have emerged.

It is noteworthy that there are still few preregistered replications of the basic MS effect. Hayes and Schimel (2018) performed a series of three experiments, where Study 2 was a preregistered online experiment. This experiment showed a decrease in self-esteem after performing a word-completion task with death related words. However, the effect only emerged when applying an unregistered exclusion of some of the participants. Pepper and colleagues (2017) failed to replicate the MS effects from a previous study (Griskevicius et al., 2011), and a recent preregistered replication of a much cited TMT experiment failed to observe any evi- dence for a MS effect (Rodríguez-Ferreiro et al., 2019). This study also provided an analysis and a discussion of the literature cited by Burke and colleagues (2010), and argued that the distribution of reported effect sizes given the sample sizes did not follow the distribution one would expect from complete reports of a true effect. This makes it an open question to what extent the MS effect can be reproduced and replicated under more restrictive and transparent conditions.

\section{Mechanisms of mortality salience}

MS effects are typically described in relatively general terms (i.e., "threshold for awareness", and "proximal and distal defences"), without going into details about the cognitive or psychophysiological mechanisms for the effects. As other priming effects, MS may be accounted for by spreading activation in semantic networks (Morewedge and Kahneman, 2010). If so, cognitive representations related to death are closely linked to representations of cultural values in an associative network, so that activation of one part of the network lowers the threshold for activating semantically linked parts of the network (see Arndt et al., 2002, for a similar account). If MS works through such a mechanism, a conceptual replication would be to expect the MS manipulation to lead to increased distraction when a Stroop task presents words related to in-group classification. This may be compared to MS studies that have used a lexical decision task and similar measures to estimate death thought accessibility (Hayes et al., 2010; Hayes et al., 2008). Further, it has been argued that MS increases "tension", "discomfort" or "reluctance" associated with being reminded of death, which proximal or distal defences may reduce (Greenberg et al., 1995; Greenberg et al., 1992). If so, one may expect the MS manipulation to lead to increased psychophysiological activation either during stimulation, or as a residual effect while the MS is in effect (see e.g. Arndt, 1999; Arndt et al., 2001; Rosenblatt et al., 1989). Previous research has suggested that MS effects may be moderated by individual differences in cognitive style (Juhl and Routledge, 2010) or political orientation (Burke et al., 2013), which would indicate that one should control for such factors or examine possible interaction effects.

\section{Generalizability of mortality salience.}

Although it has been claimed that MS is a fundamental motivator for vast aspects of the human condition (Greenberg et al., 1986; Greenberg et al., 1997), a majority of the studies $(72.6 \%$ in a meta-analysis, Burke et al., 2010) used the same outcome measures, namely an effect on attitude measures. 
Moreover, a considerable part of the MS literature has used outcome measures that may be confounded with aggression towards out-group members in the face of threats (such as increase in patriotism or support for the local sports team, see e.g. Stets, 2006; Turner et al., 1994). The TMT claim that MS increases adherence to cultural values would be better supported if it could be shown for cultural values that cannot be construed as out-group aggression.

Finally, although some cross-cultural work has been done (Heine et al., 2002; Routledge et al., 2010), studies in more diverse cultural settings are needed. Crosscultural studies could indicate the wider applicability of a MS effect, and could also contribute to exclude competing causal mechanisms. If MS effects could be shown for patriotism and other values outside of North America and Central Europe, this would further support the argument that MS enhances ingroup processes.

\section{Study overview}

As reviewed above, there are reasons to question the robustness of MS effects and the underlying mechanism. To address concerns regarding mechanism and generalizability, our Experiment 1 was done in a lab setting and included both the traditional measure of national patriotism and novel measures of democratic values, prosociality, Stroop processing and psychophysiology. To address the need for high-powered direct replications, Experiment 2 was conducted as an online experiment of MS effects on a measure of national patriotism, with additional measures of in-group favouritism and prosociality. The sample size for main effects in Experiment 1 is about twice as large as the typical study in the published MS literature, whereas the sample size in Experiment 2 is over 17 times larger than the typical MS study (calculated from the $\mathrm{n}$ per analysis cell reported in the meta-analysis of Burke et al., 2010). This provides sufficient power for both experiments to detect the effects reported in the literature. Power to detect effects of different sizes are discussed in a later section. All hypotheses and analysis approaches for both experiments were preregistered ahead of data collection and were performed in accordance with local ethical guidelines.

\section{Experiment 1 background}

Based on the above review, the main aim of Experiment 1 was to replicate the traditional MS effect on national patriotism in a lab setting. As further aims, we also attempted to conceptually replicate the MS effect in novel but theoretically related outcome measures, while controlling for individual cognitive and psychosocial sensitivity to the manipulation. Our preregistration (available at https://osf.io/ec4yk) described four manipulation checks and six hypotheses, which are justified below and listed in Table 2. ${ }^{1}$

To test that the construct of mortality interferes with cognitive processing, we checked (E1MC1) whether the MS group were slower to respond to deathrelated words. To assess whether we succeeded in manipulating MS on a psychophysiological level, we checked (E1MC2) whether the MS group had higher psychophysiological activation. Further, we checked that (E1MC3) the pro-patriotic was preferred over the anti-patriotic essay, and whether (E1MC4) the pro-democratic essay was preferred over the antidemocratic essay. In order to directly replicate the most common type of MS research where MS increases preference for national patriotism, Experiment 1 measured preference for pro and anti-Norway essays (essays were taken from Rosenblatt et al., 1989, with minor cultural adjustments). Here, we expected (E1H3) participants in the MS group to show a higher preference for the essay expressing national patriotic values.

Data was collected among a Norwegian population where democratic values of privacy, citizenship and human rights are mainstream pro-social values. On this background, the patriotic essays were supplemented with essays about how the concern for democratic values should be handled in the aftermath of a terrorist attack. If MS increases the relevance of ingroup cultural values, one would expect (E1H2) an effect of increased preference for an essay expressing democratic values, compared to an essay expressing anti-democratic values. The novel essays were included to test whether MS effects could be shown to be independent of outgroup aggression.

A possible underlying mechanism of MS may be that existential threats make membership to social groups more important, and thus make people more aware of social categorization. If so, we would expect (E1H1) that MS activates cognitive constructs related to "social categorization", and thus makes these words more intrusive on Stroop processing, resulting in longer response times ("RT").

While most MS studies measure attitudes (using essays like the types mentioned above), it would be beneficial to supplement this with measures of behavioural intentions. Previous research on charitable giving has suggested that people are more generous towards recipients that belong to a common ingroup (Everett et

\footnotetext{
${ }^{1}$ The study was preregistered before any of the data was inspected. Please note that different dates may be displayed for the preregistration of Experiment 1. The correct timestamp is $2015-10-12$, as shown in the OSF registration date and the OSF version control.
} 
al., 2015; Grimson et al., 2020). Some studies have found MS to increase donation to charities (Jonas et al., 2002; Roberts and Maxfield, 2019; Zaleskiewicz et al., 2015). We included a novel measure of pro-sociality that asked participants how they would have shared a hypothetical lottery prize with individuals that were closely or more distantly related to them. This was intended as a short online measure that could also distinguish between charity towards different groups, in order to better explore the motivation for the charity (i.e., giving to family and relatives, as opposed to giving to strangers). This measure was partly inspired by Singer's (2011) concept of a "moral circle" that may include people more or less "distant" from you. The structure of the task is very similar to the "dictator game" (Kahneman et al., 1986), where the decision-maker is asked to divide a given endowment between themselves and an anonymous partner, which is an established choicemeasure of generosity that has previously been adapted to charity giving as well (see Sjåstad, 2019). In the TMT account, one would expect (E1H4) the MS group to express a preference for sharing more of the prize with non-relatives, in an attempt to be remembered beyond their physical death by a larger social circle. ${ }^{2}$

Previous studies have claimed that MS effects are more evident in more cognitive flexible individuals (Juhl and Routledge, 2010). We thus expect (E1H5) the MS effects on the four measures listed above to be enhanced for participants low on "need for closure" (NfC, Federico et al., 2007). Finally, one may expect individual differences in the effectiveness of the MS stimulation, and that this may be indexed by psychophysiological measures. Heart rate variability (HRV, Acharya et al., 2006) may be used as an index of the body's ability to adapt to the changing demands of the environment. Higher HRV (more high-frequency modulation of heartbeat intervals) has been taken to indicate more physiological adaptability and executive function, while lower HRV has been argued to indicate states of stress and emotional activation (Delaney and Brodie, 2000; Lane et al., 2009). The HRV is an unobtrusive measurement after it has been mounted. As the TMT account states that MS leads to an uncomfortable emotional state that is alleviated through either proximal or distal defences. We thus expect (E1H6) the MS effects on the four measures listed above to be enhanced for participants with lower HRV. See Table 1 for an overview of manipulation checks and hypotheses.

The TMT literature emphasizes the need for a "delay task" to avoid conscious processing of the death reminder (although delay tasks are not used consistently, see Burke et al., 2010). No explicit delay task was used Experiment 1, to avoid the risk of fatigue effects of an overly long experiment obscuring any MS effects. However, there were two tasks not related to mortality following the traditional MS manipulation and preceding the traditional MS measurement (experiment procedure in next section). In particular, the "social Stroop task" may serve the role of a delay task that precedes all outcome measures.

\section{Experiment 1 methods}

\section{Experiment 1 outline}

We conducted a laboratory experiment where the predictor variable was MS vs. control manipulation (between subjects, two conditions). The outcome variables were RT for death-related words, RT for social words, preference for pro-democratic essays, preference for pro-patriotic essays, NfC and HRV. The preregistered experiment procedure and materials can be found online at https://osf.io/naxz6/. Data was collected in our lab between 2015-09-29 and 2015-10-30.

\section{Experiment 1 sample}

We recruited 101 university students (44 female) through email advertising (preregistered sample was 100 , one participant was replaced during data collection due to non-compliance). This sample size was set in order to be larger than the typical MS experiment in the published literature, while also restricted by practical concerns for in-lab studies. Please see the full power analysis in the Discussion. All participants were undergraduate psychology students, self-identified as having a Norwegian identity and normal colour vision. Median age group was 22-25 years old. The experiment program randomized participants to the MS group or the control group, without the experimenters knowing who were in which group. Due to an administration error, there were 52 participants in the MS group and 48 participants in the control group. No participants were excluded in the data analysis.

\section{Experiment 1 procedure}

The whole experiment took about 25 minutes, and was conducted in sound attenuated testing booths, on desktop computers running the E-Prime experiment presentation software (Psychology Software Tools,

\footnotetext{
${ }^{2}$ Note that the preregistrations distinguished between a E1H4a and E1H4b. E1H4b was based on the theoretical framework of coalition psychology, and would be supported if MS group participants expressed a greater preference to share the prize with genetically related individuals. We have excluded this discussion since the distinction between the two accounts is unclear and neither of them was supported.
} 
2012), where responses were given with a PC keyboard. After signing an informed consent form, all participants went through the following experiment procedure:

1. A seven-item scale for «Need for closure» (about 1 minute).

2. MS manipulation: Two questions where participants were asked to write short responses about either «death» or «toothache» (2-3 minutes).

3. A «social Stroop» task with half the words related to social categorization (about 5 minutes).

4. Pro- and anti-democratic essays (order counterbalanced), each followed by five questions evaluating the essay's content and author (about 5 minutes).

5. Pro- and anti-patriotic essay (order counterbalanced), each followed by five questions evaluating the essay's content and author (about 4 minutes).

6. Pro-sociality measure (1-2 minutes).

7. A «death Stroop» task with half the words related to death (2-3 minutes).

In the beginning of the experiment (stage 1), participants filled out a questionnaire for cognitive style, using a seven item measure of NfC (Federico et al., 2007, translated to Norwegian by the authors).

For the manipulation (stage 2) participants were randomized into two experimental groups, which were asked two different questions. The randomization was done by the experiment presentation software and was double blinded for both participants and experimenters. We used the traditional manipulation of MS (Rosenblatt et al., 1989), where both groups were asked to write down their answers to two short questions presented sequentially, using the standard instructions of «responding based on gut feeling». The first question asked about thoughts and feelings evoked by «death» for the MS group (or «toothache» for the control group), while the second question asked about what they thought will happen at death and after death (or toothache). This manipulation was used in $79.8 \%$ of the MS studies in a meta-analysis (Burke et al., 2010). A manual inspection showed that all participants provided relevant answers.

A Stroop task was presented (stage 3), with words written in red, blue, green or yellow text against a grey background, and participants were asked to indicate what colour each word was written in as quickly as possible. $50 \%$ of the words were related to social categorization (such as «them», «us», «conflict» and «cooperation»), and $50 \%$ neutral words matched for letter length and word frequency. There were 200 trials, and the order of presentation for the words was online randomized. Participants responded by using mouse clicks, where the placement of the response boxes switched between trials (this was done to avoid preference for left/right side or centre/lateralized responses, and thus each trial required a visual search for the intended response). For each participant, we calculated the ratio of time taken to respond to social words divided by the time on neutral words. Only responses with RT within one SD of the participant's mean were included in the analysis (applying a more lenient criteria of two SD did not significantly change the results).

Next (stage 4 and 5), four brief essays were presented. The first two essays (about 200 words long) were novel for the study and presented two opposing views of how Norwegian security policy should be handled in the aftermath of a terrorist attack that happened four years before the data collection. The themes of these essays were whether Norwegian society was essentially safe or under threat, whether extreme viewpoints should be discussed in public or censored, whether the best safeguard against terrorism is social integration and prevention or surveillance and control, and whether terror measures should be balanced against democratic rights or not. The next two essays (about 120 words long) were pro- and anti-patriotic essay that are traditionally used in MS studies (Rosenblatt et al., 1989). In the review of MS experiments Burke et al. (2010) a wide range outcome variables are used, but the most commonly used is that MS leads to a more favourable evaluation of national patriotic essays (and less favourable evaluation of non-patriotic essays). The essays had been translated into Norwegian by the authors and one aspect changed to suit the Norwegian setting (from "picking fruits" to "work as store clerk"). All four essays are available online both in Norwegian as they were used in the experiment and translated to English (https://osf.io/d2zus/). Whether the pro or anti essays were presented first was counterbalanced between participants. After each of the four essays, participants were asked to rate on a nine-point scale how well they liked the author, how intelligent and knowledgeable they thought the author was, to which extent they agreed with the essay and thought it made an accurate assessment of the issue (higher scores indicating a more positive evaluation). Essay evaluations were calculated as the participant's average score for the answers to the five questions for each of the four essays (Cronbach's alpha for pro-democratic essays $=$ .92 , for anti-democratic essays $=.93$, for pro-patriotic essay $=.89$, for anti-patriotic essay $=.91$ ). For each participant, a difference score was calculated between 
the responses to the pro- and the anti-patriotic essays, and similarly for the democratic essays (for both difference scores higher values indicated being more positive to the pro than the anti essays). ${ }^{3}$

Thereafter (stage 6), a novel measure of pro-sociality was applied, where the participants were asked how they would have liked to share a hypothetical lottery prize equivalent to about USD 1.000.000 between their (a) close family (parents and siblings), (b) relatives (grandparents, uncles and aunts, cousins), (c) friends and (d) with charities, and (e) what they would keep for themselves and any immediate family. Participants typed in a percentage for how much they wanted to share with each party. All the entered percentages were displayed on screen. The participants were asked to check if they were satisfied with the distribution and had the option to distribute again. A ratio was calculated of the percentage assigned to the latter two recipients (friends and charity) over the percentage kept for self, family and relatives $(c+d) /(a+b+e)$.

Finally (stage 7), a second Stroop task (92 trials) was presented, where $50 \%$ of the words were related to mortality (e.g., «funeral», «obituary» and «mortal»), with matched neutral words. Presentation, response and calculations were similar as in the first Stroop task (stage 3). This stage was placed last in the experiment, to prevent the presentation of the death-related words to interfere with the assumed MS effect on previous stages. This is in line with the concern of Hayes and Schimel (2018) that activation of death-related constructs may disrupt the measurement of MS effects.

Throughout the experiment, heart rate was measured from all participants using Polar RS800cx waist sensor and wrist recording unit. We excluded HRV data from participants with signal loss for more than $1 / 3$ of the recording. We had a preregistered approach to select a 5-minute analysis window from the middle of the recording (which roughly matches when participants evaluate the essays), and the onset was adjusted based on data quality in the window (before identifying participants to their experimental condition). HRV was calculated as the root mean sum of squared differences (RMSSD) of the distance between the peak of each QRS complex (RR beat-to-beat interval, a timedomain analysis). A conventional interpretation (Delaney and Brodie, 2000) is that lower HRV indicates states of stress and emotional activation (but note that this interpretation has been disputed).

\section{Experiment 1 results}

Based on our planned directional predictions, we performed one-tailed null hypothesis testing of our manipulation checks E1MC1, E1MC2, E1C3, E1C4 and hy- potheses E1H1, E1H2, and E1H3. As the direction of the hypothesis E1H4 for the pro-sociality task was not clear in the preregistration, we tested it with a twotailed test. All analyses were done in the jamovi software (jamovi project, 2019). See Table 1 for a summary of manipulation checks, hypothesis testing and results. Dataset (https://osf.io/2q7kp/) and analyses (https://osf.io/n6ysk/) are available online.

\section{Manipulation check}

The first manipulation check (E1MC1) gave no indication that the semantic construct of «death» was more central for the MS participants. Neither did the second manipulation check (E1MC2) show a significant effect of MS on psychophysiological activation (HRV). An additional test of HRV at the time of the MS manipulation also failed to find a significant effect of MS (one-tailed $p$ $=.081$ ). The remaining manipulation checks (E1MC3 and E1MC4) showed that the intended essay was preferred in both pairs of essays (pro-Norway and prodemocratic values, respectively), confirming that the essay measure was successful at creating a cultural ingroup versus outgroup scenario.

\section{Confirmatory analyses}

The planned tests of E1H1, E1H2 and E1H3 were performed with one-tailed t-tests in the direction stated in the preregistration. Each were followed up with General Linear Model analyses with NfC score or HRV score was included as covariates to test for interaction effects (respectively corresponding to E1H5 and E1H6 hypotheses in the preregistration). This approach is functionally equivalent to the GLM approaches described in the preregistration. The planned tests for $\mathrm{E} 1 \mathrm{H} 1$ showed no significant main effect of MS on the «social Stroop» test, nor any significant interaction with NfC or HRV. E1H2 showed no significant main effect of MS on rating of democratic essays, nor any significant interaction with NfC or HRV. E1H3 showed no significant main effect of MS on the rating of patriotic essays, nor any significant interaction with NfC or HRV. Answer distributions on this central outcome variable is shown in violin plots in Figure 1, indicating no difference in distribution between the experiment conditions. Thus, Experiment 1 did not find any support for the primary hypotheses of the MS effects found in the TMT literature.

\footnotetext{
${ }^{3}$ This pre-registered approach is consistent with the standard method in previous research on TMT. Open data and code are provided in order for interested readers to perform alternative analyses (e.g., using the pro-essay score as a covariate for the effect on the anti-essay score).
} 
Table 1

List of manipulation checks and hypotheses, operationalization, tests and extent of support in Experiment 1 (one-tailed p-values where not otherwise indicated).

\begin{tabular}{|c|c|}
\hline Name & Operationalization \\
\hline $\begin{array}{l}\text { E1MC1: MS will make } \\
\text { semantic constructs re- } \\
\text { lated to death more ob- } \\
\text { trusive on reading. }\end{array}$ & $\begin{array}{l}\text { The MS group will } \\
\text { show longer RT for } \\
\text { reporting the colour } \\
\text { of words with «death } \\
\text { related» content com- } \\
\text { pared to words with } \\
\text { neutral content. }\end{array}$ \\
\hline
\end{tabular}

E1MC2: $\quad$ MS will Participants in the MS increase psychophysio- group will have lower logical activation

E1MC3: The pro- Participants will show democratic essay a preference for the represents the majority pro-democratic essay. view

E1MC4: The pro- Participants will show patriotic essay rep- a preference for the resents the majority pro-patriotic essay. view

E1H1: MS will increase activation of semantic constructs related to social categorization

Participants in the MS group will have longer RT to the social words than to the neutral words in a Stroop task.
Statistical test One-tailed t-test of difference between conditions in ratio of death-word RT to neutral word RT.

One-tailed t-test of RMMSD difference between conditions during essay reading.

One-tailed t-test will show a higher average score on five questions about the prodemocratic essay than average of same questions for the anti-democratic essay.

One-tailed t-test will show a higher average score on five questions about the propatriotic essay than average of same questions for the anti-patriotic essay
A t-test of experiment group (MS group vs. control group) on Stroop effect for social words as outcome variable. To test the H5a prediction, the same relationship was also tested with a two-way ANOVA with an added interaction of $\mathrm{NfC}$ score. As predicted by $\mathrm{H6a}$ prediction, the same relationship was also tested with a two-way ANOVA with an added interaction HRV index.
Results

Not supported $(\mathrm{N}=99$, MS group $\mathrm{M}=11.9 \mathrm{~ms}(\mathrm{SD}=$ 66) vs. control group $\mathrm{M}=$ $7.2 \mathrm{~ms}(\mathrm{SD}=48.3), p=$ $.345, \mathrm{CI}=$-inf. $-14.7, \mathrm{~d}=$ $-0.08)$.

Not supported ( $\mathrm{N}=75$, MS group $\mathrm{M}=44.7(\mathrm{SD}=19.9)$ vs. control group $\mathrm{M}=54.1$ $(\mathrm{SD}=31.7), p=.065$ in expected direction, $\mathrm{CI}=-0.8-$ inf., $d=0.35)$.

Supported $(\mathrm{N}=100$, proessay $\mathrm{M}=6.7(\mathrm{SD}=1.3)$ vs. anti-essay $\mathrm{M}=3.7$ (SD $=1.5), p<.001$ in expected direction, $\mathrm{CI}=2.73$ - inf., $\mathrm{d}$ =1.64).

Supported $(\mathrm{N}=100$, proessay $\mathrm{M}=6.5(\mathrm{SD}=1.25)$ vs. anti-essay $\mathrm{M}=4.1$ (SD $=1.5), p<.001$ in expected direction, $\mathrm{CI}=2.08-$ inf., $\mathrm{d}$ = 1.37).

Not supported ( $\mathrm{N}=99$, MS group $\mathrm{M}=-2.3 \mathrm{~ms}(\mathrm{SD}=$ 40.8 ) vs. control group $M$ $=11.6 \mathrm{~ms}(\mathrm{SD}=58.5), p=$ $.09, \mathrm{CI}=$ inf. $-30.7, \mathrm{~d}=$ .28). E1H5a not supported (interaction $p=.44$ ). E1H6a not supported (interaction $p$ $=.55$ ). 
E1H2: MS will in- Participants in the crease support for MS group will show democratic values a higher preference for the democratic essay compared to the anti-democratic essay.

E1H3: MS will in- Participants in the crease national patrio- MS group will show tism

E1H4: MS will affect the degree of prosociality

The amount shared with friends and charities relative to the
A t-test for experiment group (MS group vs. control group) on pro/anti-democratic essays as outcome variable. To test the $\mathrm{H} 5 \mathrm{~b}$ prediction, the same relationship was also tested with a two-way ANOVA with an added interaction of NfC score. To test the $\mathrm{H} 6 \mathrm{~b}$ prediction, the same relationship was also tested with a two-way ANOVA with an added interaction of HRV index.

A t-test for experiment group (MS group vs. control group) on patriotic essays as outcome variable. To test the $\mathrm{H} 5 \mathrm{c}$ prediction, the same relationship was also tested with a two-way ANOVA with an added interaction of NfC score. To test the H6c prediction, the same relationship was also tested with a two-way ANOVA with an added interaction of HRV index. amount shared with family will be different for the MS group.
Not supported ( $\mathrm{N}=100$, MS group $\mathrm{M}=3.1(\mathrm{SD}=1.9)$ vs. control group $\mathrm{M}=2.9$ $(\mathrm{SD}=1.8), p=.29, \mathrm{CI}=$ -inf. $\quad-0.41, d=-0.11)$. E1H5b not supported (interaction $p=.87$ ). E1H6b not supported (interaction $p=$ .45).

Not supported ( $\mathrm{N}=100$, MS group $\mathrm{M}=2.3(\mathrm{SD}=1.75)$ vs. control group $\mathrm{M}=2.4$ $(\mathrm{SD}=1.73), p=.58, \mathrm{CI}=$ -inf - 0.65, $d=.04)$. E1H5c not supported (interaction $p$ $=.85)$. E1H6c not supported (interaction $p=.29$ ).

Not supported $(\mathrm{N}=100, \mathrm{M}$ $=0.44(\mathrm{SD}=0.71)$ vs. $\mathrm{M}=$ 0.25 (SD = 0.31), two-tailed $p=.09, \mathrm{CI}=$-inf. --0.01 , $\mathrm{d}=0.35)$. E1H5d not supported (interaction $p=.22$ ). E1H6d not supported (interaction $p=.99$ ). 


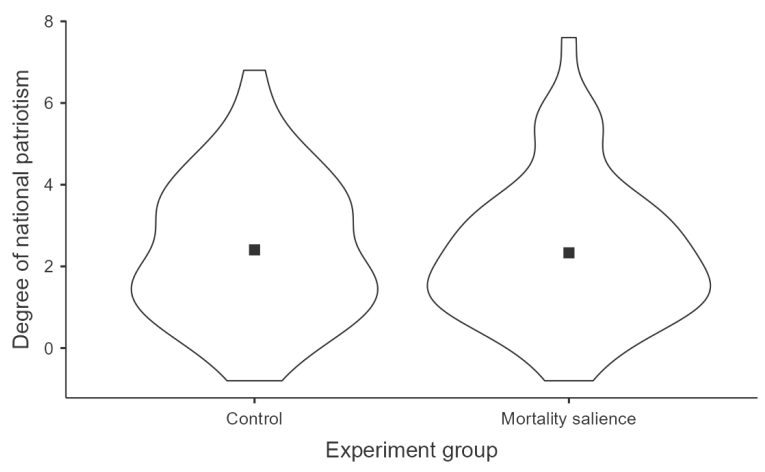

Figure 1. Violin plot of scores on patriotic essay evaluation difference score in Experiment 1, which did not show a significant difference between mortality salience and the control condition ( $\mathrm{N}=100$, Norway).

On the measure of pro-sociality, the MS group stated they would share more $(23.1 \%)$ with friends and charities than the control group (16.9\%). An effect in that direction would support the E1H4 prediction derived from TMT that MS should increase preference for sharing with non-relatives. However, the effect was not significant in the two-tailed test prescribed by the bidirectional hypothesis in the preregistration. The score distributions indicate that the difference may have been driven by a few extreme values (see violin plot in analysis files on OSF). Nevertheless, the difference in means between the groups justifies a further examination of this measure, which is performed in Experiment 2. There were no significant interactions of NfC or HRV on the pro-social task.

\section{Experiment 2 background}

The main aim of Experiment 2 was to directly replicate the traditional effect of MS on national patriotism in an American sample. As additional aims we wanted to test conceptual replication of MS effects on ingroup identification and pro-sociality. The preregistration for Experiment 2 is available at https://osf.io/d26fq.

As manipulation checks, we (E2MC1) verified that the pro-USA essays were in fact preferred over the antiUSA essays. In addition, we (E2MC2) manually verified that participants had in fact provided meaningful answers to the manipulation questions about death (preregistration of this analysis: https://osf.io/sw6md).

The first hypothesis (E2H1) tested whether MS effects involve mechanisms of ingroup identification and group membership. This corresponds to the mechanism tested in E1H1 in Experiment 1 (the "social Stroop test"), but the current test has higher face-validity. Moreover, the test is an attempt to replicate an effect of
MS leading to higher ingroup identification previously found in an Italian sample (Castano et al., 2002).

The second hypothesis (E2H2) was intended to directly replicate the traditional lab experiments, where participants who write short answers to questions about death showed increased patriotism later in the study (Greenberg et al., 1994; Greenberg et al., 1992; Simon et al., 1997). We attempted to make both manipulation and effect measure in the online experiment as similar as possible to the traditional experiments.

The third hypothesis (E2H3) was intended to further explore the effects of MS on the novel measure of prosociality used in Experiment 1. This measured generosity towards people outside your family, and is thus an indicator of pro-sociality. Previous studies have shown MS to lead to increased pro-sociality (Jonas et al., 2002; Roberts and Maxfield, 2019; Zaleskiewicz et al., 2015).

While Experiment 1 had no explicit delay task, to maintain similarity with the research we want to replicate, Experiment 2 included a 20-item mood measure to serve this function. The same delay task is often used in the TMT literature. The delay task was presented after the MS manipulation, and before the three outcome measures. Finally, we also measured political orientation in order to perform a preregistered exploration of whether political orientation moderates the predicted effect of E2H1, E2H2 or E2H3.

\section{Experiment 2 methods}

\section{Experiment 2 outline}

We conducted a high-powered online experiment on a total sample of 800 US participants. As in Experiment 1 , the predictor variable was MS versus control. The outcome variables were American ingroup identification, national patriotism, and pro-sociality.

\section{Experiment 2 sample}

A total of 803 US participants signed up for this study in exchange for \$ 0.50 USD. After excluding 19 duplicate and one incomplete responder, the final sample consisted of 784 participants (389 randomised to the MS condition, age $\mathrm{M}=38,61 \%$ female). As noted earlier, this sample is more than 17 times larger per analysis cell than the typical MS experiment in the TMT literature. See power-curve in Discussion. Data was collected on July 12th, 2019.

\section{Experiment 2 procedure}

The Amazon Mechanical Turk online platform (Buhrmester et al., 2011; Hauser and Schwarz, 2016) 
was used to recruit participants for a study about "personality and attitudes", and the experiment was programmed and administered on the Qualtrics platform (available as online materials: https://osf.io/jm4uh/). On average participants spent slightly over 8 minutes on the experiment. The experiment procedure was as follows:

1. MS manipulation: Two questions where participants were asked to write short responses about either «death» or «toothache», (2-3 minutes).

2. Delay task: Indicate current mood, 20 questions (about a minute).

3. DV1: Ingroup identification, 5 questions (about half a minute).

4. DV2: National patriotism, ratings of pro-USA and anti-USA essays, order counterbalanced (about 3 minutes)

5. DV3: Pro-social task (1-2 minutes).

6. Moderator: variable: Political orientation (a few seconds).

When clicking through to the survey, participants were randomized to receive the MS manipulation or the control task. At the beginning of the experiment (stage 1 ), the MS and control group were asked to write brief answers to the same two questions as in Experiment 1 about either "death" or "toothache", respectively. The two questions were presented on separate pages, and answers were written in an empty text box beneath each question. To avoid that participants could simply click their way through the survey without responding, the answer to each of the two questions had to be at least 15 characters long before they could proceed. While this approach to a manipulation check has weaknesses, it should be noted that manipulation checks even to this extent are rarely reported in the published MS literature. An advantage of this manipulation check is that the subsequent assessment of written responses should not interfere with the subconscious processing of death reminders that is proposed by TMT.

On the next screen (stage 2), there was a delay task of answering 20 questions about current mood (PANAS; Watson et al., 1988). Participants were asked to rate the extent they felt "interested", "distressed", "excited" etc. on a five-point scale from "Not at all" to "Extremely". This task was included to maintain similarity to traditional MS experiments. The same task is used in $47.7 \%$ of the MS literature (Burke et al., 2010), most typically as the only delay task.. The inclusion of a delay task between the manipulation and the outcome variables is sometimes argued to be critical for the MS phenomenon to emerge.

Thereafter (stage 3) participants were presented with a screen with five statements about their American identity, based on the group identification scale (Doosje et al., 1995). Each item was rated on a seven-point scale from "Not at all" to "Totally". The statements were: "I perceive myself as an American", "I feel strong ties with other Americans", "Being an American does not mean much to me" (reversed), "I identify with American people", and "Being an American has nothing to do with my identity" (reversed). Cronbach's alpha for the responses was .84.

Next (stage 4), national patriotism was measured with one pro-USA and one anti-USA essay presented sequentially in counterbalanced order. These were the traditional essays used in MS experiments (see e.g. Rosenblatt et al., 1989) and similar to the essays used in Experiment 1 , except that they had not been translated and adapted to fit a Norwegian context. After reading each essay, participants answered the same five questions as in Experiment 1 (Cronbach's alpha $=.91$ for pro-USA, .95 for anti-USA). To get a score for national patriotism, the average score on the anti-USA essay was subtracted from the average score on the pro-USA essay (higher scores indicate higher patriotism).

Thereafter (stage 5), the same pro-social task as in Experiment 1 was applied (value of lottery win set to USD 1.000.000). In a sequential list, participants entered the percentage they would like to share with (a) self or immediate family, (b) close family, (c) extended family, (d) friends, and (e) charities. The summed percentage was shown beneath, and the sum had to be $100 \%$ in order to continue the experiment. As in Experiment 1 and as preregistered, an index for pro-sociality was calculated as $(d+e) /(a+b+c)$, in which a higher number indicates a higher level of pro-sociality.

At the end of the experiment (stage 6), participants reported age and gender. Then as a single-item measure of political views the question "In general, what would be the most accurate description of your political views?" was answered on a 7-point scale (marked with $1=$ Very leftwing/ liberal, 4 = centrist/ moderate, 7 = Very rightwing/ conservative). The responses used the full range, with a central tendency in the middle $(\mathrm{M}=3.64, \mathrm{SD}=$ 1.8).

\section{Experiment 2 results}

The manipulation checks confirmed that the pro-USA essay was preferred over the anti-USA essay (E2MC1), indicating that national patriotism as expressed in these essays was in fact the dominant cultural value. Further, almost all participants did in fact write meaningful re- 
Table 2

List of manipulation checks and hypotheses, operationalization, tests and extent of support in Experiment 1 (one-tailed p-values where not otherwise indicated).

\begin{tabular}{ll} 
Name & Operationalization \\
\hline E2MC1: The pro-USA & Participants will show \\
essay will be preferred & a preference for the \\
over the anti-USA es- & pro-patriotic essay. \\
say (across conditions) &
\end{tabular}

E2MC2: The manipulation instructions were followed

E2H1: MS will increase ingroup identification
Participants will provide meaningful answer to the manipulation questions

Participants in the MS group will have to a larger degree identify as Americans.
E2H2: MS will increase national patriotism

E2H3: MS will increase pro-sociality
Participants in the MS group will show a higher preference for the patriotic essay compared to the anti-patriotic essay.
A t-test for experiment group (MS group vs. control group) on patriotic essays as outcome variable. Regression of political views on patriotism. Moderation of political views on relationship between mortality salience and patriotism.

Participants in the MS group will state that they would share more of a hypothetical money prize with friends and charities relative to the amount shared with family and relatives.

A t-test for experiment group (MS group vs. control group) on the ratio of giving to friends and charities as outcome variable. Regression of political views on pro-sociality. Moderation of political views on relationship between mortality
Statistical test

One-tailed t- $\neg$ test will show

a higher average score on five questions about the propatriotic essay than average of same questions for the anti-patriotic essay.

Manual classification of all 800 responses

A t-test of experiment group (MS group vs. control group) on ingroup identification score. Regression of political views on ingroup identification. Moderation of political views on relationship between mortality salience and ingroup identification.

Results

Supported $(\mathrm{N}=784, \mathrm{MS}$ group $\mathrm{M}=5.4(\mathrm{SD}=1.04)$ vs. control group $\mathrm{M}=4.4$ $(\mathrm{SD}=1.62), p<.001$ in expected direction, $d=0.59$ ).

98\% provided relevant responses

Not supported $(\mathrm{N}=784$, MS group $\mathrm{M}=5.2(\mathrm{SD}=1.38)$ vs. control group $\mathrm{M}=5.28$ $(\mathrm{SD}=1.39), p=.46, \mathrm{~d}$ $=0.05$ ). More conservative participants showed significantly higher ingroup identification $(\mathrm{t}=9.67, p<.001)$. No significant moderation of political views $(\mathrm{Z}=-0.8, p=$ .425).

Not supported ( $\mathrm{N}=784$, MS group $\mathrm{M}=1.15(\mathrm{SD}=1.99)$ vs. control group $\mathrm{M}=1.17$ $(\mathrm{SD}=1.95), p=.91, \mathrm{~d}$ $=0.01$ ). More conservative participants showed significantly higher patriotism $(\mathrm{t}=$ $11, p<.001)$. No significant moderation of political views on patriotism $(\mathrm{Z}=-0.04, p$ $=$.97). salience and patriotism.
Support for less sharing in MS group $(\mathrm{N}=781, \mathrm{M}=$ 0.177 (SD $=0.267)$ vs. control group $\mathrm{M}=0.234$ (SD = 0.234), $p=.036, \mathrm{~d}=.15$ ). No significant effect of political views on pro-sociality $(\mathrm{t}$ $=1.62, p=.106)$. No significant moderation of political views on sharing $(\mathrm{Z}=1.5, p$ $=.135)$. 
sponses to the MS experimental manipulation (E2MC2), indicating that the manipulation was successful in evoking thoughts about death versus toothache (control). Human verification of written responses (E2MC2) also helped ensure the data quality, since participants randomly clicking their way through the survey or not understanding the instructions would be screened out in this procedure. The written responses are described in more detail in a separate publication (Storelv and Sætrevik, 2021).

In accordance with the preregistration we tested $\mathrm{E} 2 \mathrm{H} 1, \mathrm{E} 2 \mathrm{H} 2$ and $\mathrm{E} 2 \mathrm{H} 3$ as t-tests against a two-tailed p-value of .05 . In addition, we tested the effects of political orientation on the outcomes as simple regressions, and we tested their moderating effect on the relationship between MS and outcomes. All analyses were done in the jamovi software (2019), using the "medmod" module for the moderation analyses. See Table 2 for results from the preregistered confirmatory hypotheses tests (E2H1, E2H2 and E2H3). Dataset and analyses are available online (at https://osf.io/mejnt/ and https://osf.io/zpn92/).

Despite the successful manipulation check, the results showed no significant difference between the MS and the control group on ratings of ingroup identification (E2H1). Further, there was no significant difference between the MS and control group on the focal outcome measure of national patriotism (E2H2). A violin plot of the scores is shown in Figure 2. Thus, Experiment 2 did not show any support for the primary hypotheses about the effect of death reminders, as derived from TMT and previous research. On the pro-sociality measure there was a small but significant difference in the opposite direction of the $\mathrm{E} 2 \mathrm{H} 3$ prediction $(\mathrm{d}=.15, p=.036)$, of the MS group sharing less ( $12.7 \%$ of the amount) than the control group (14.8\%) with friends and charities.

As suggested in the preregistration, we explored the possible moderator effect of political orientation on the outcome variables. Political orientation was significantly correlated with two of the outcome measures, indicating that more conservative participants identified more as Americans and showed higher national patriotism, while there was no significant effect on prosociality. However, there was no significant interaction between MS and political orientation on neither of the three outcome measures (ingroup identification, national patriotism and pro-sociality), thus showing no difference in how conservative and liberal participants responded to the MS manipulation.

\section{Discussion}

The aim of the current study was to directly replicate the effect of MS on attitudes to national patriotic essays,

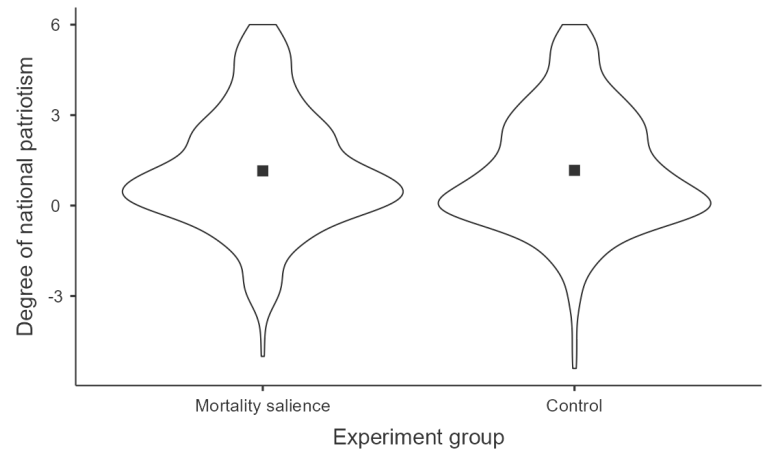

Figure 2. Violin plot of difference in national patriotism between experiment groups in Experiment 2, which did not show a significant difference between mortality salience and the control condition ( $\mathrm{N}=784$, USA).

and to conceptually replicate the effect on other measures to explore mechanisms and boundary conditions. This was tested across two preregistered experiments, one in a lab and one online, with a combined sample of 884 participants from two different countries. Despite our best efforts, we failed to both directly and conceptually replicate the MS effects. Neither did we find indications of the assumed mechanism of MS (word processing times, psychophysiology or ingroup identification). The second experiment showed a small but significant effect on pro-sociality, where MS led to reduced prosociality. This effect is in the opposite direction from the prediction derived from traditional MS theories and previous research. We were thus unable to obtain any empirical support for direct or conceptual replication of the MS effect or its assumed mechanisms on any of the outcome measures. As opposed to most previous research on MS and TMT, in both experiments we manually verified that the manipulation was adhered to (that the MS group in fact wrote relevant answers related to "death" themes), and provide this and all other outcomes in public datasets. We thus see it as unlikely that the null-results can be explained by a failure to manipulate death awareness. The results are further discussed below.

\section{No direct replication of MS effect on patriotism}

Both experiments failed to directly replicate the traditional MS effect on national patriotism, using the typical essay measure in both a Norwegian (E1H3) and an American sample (E2H2). Although our manipulation checks confirmed that the patriotic essay reflected the dominant cultural values in our samples, the MS treatment did not increase this preference. This result opposes much of the published MS literature, typically de- 
scribed in terms of the TMT framework (Burke et al., 2010; Greenberg et al., 1994).

Since Experiment 1 aimed for conceptual rather than direct replication, a Stroop task and a novel essay task was performed before the patriotic essay. The presence of these tasks may be a possible explanation of the nullfinding (for E1H3), although we assumed that these measures would be sufficiently indirect to not interfere with the MS effect on patriotic essays. Nevertheless, the high-powered Experiment 2 had the patriotic essays immediately after the delay task (E2H2), which would constitute a direct replication of the prototypical MS study (see e.g. Greenberg et al., 1994).

\section{No conceptual replication of MS effect on novel es- says}

For Experiment 1 we constructed novel essays for measuring MS effects on preferences for democratic values. A manipulation check (E1MC3) confirmed that the pro-democratic essay expressed values that were dominant in the sample. Our theoretical extension (conceptual replication) of the traditional MS effect was to expect that MS would increase the preference for expressing democratic values. However, the E1H2 test did not show a MS effect of increased preference for the democratic essay.

Experiment 1 thus failed to demonstrate the MS effect to transfer to a novel and culturally adapted measure (support of democratic values). Although this replication in Experiment 1 contains novel aspects, the literature often describes MS effects as being universal across cultures, and that the effect has wide-reaching consequences for most aspects of human social life. Further, reviews have shown MS effects in a wide range of outcome variables (Burke et al., 2010), and has been shown to transfer to defence of cultural values in both American, European and non-Western societies (e.g. Heine et al., 2002; Routledge et al., 2010).

\section{No direct replication of MS effect on ingroup identi- fication}

As a straight-forward test of the mechanism assumed to cause the MS effect, Experiment 2 (E2H1) tested whether the manipulation increased social identification with the larger ingroup (i.e., being an American). There was no significant effect on this measure, thus failing to support what the TMT has claimed is a fundamental mechanism behind MS effects. This also constitutes a failed replication of the results from a previous study in Italy, using similar measures (Castano et al., 2002). To our knowledge, no other studies have directly tested this assumed mechanism of MS on ingroup iden- tification but have instead tested the effect that MS has on expressing the ingroup's values.

\section{No effect of MS on word processing speed}

There was no significant effect in the Experiment 1 manipulation check of MS increasing Stroop processing times for death-related words (E1MC1). In our view, this fails to support the claims of TMT (see e.g. Arndt et al., 1997), as there was no indication that the MS manipulation made concepts related to mortality more accessible for the participant in a subsequent task.

Neither did Experiment 1 find a MS effect on Stroop processing times for words associated with social categorization (E1H1). This opposes the expectation derived from TMT that MS makes social identification (or cultural belonging) more relevant as a way of finding meaning beyond physical death. We propose three possible explanations for the lack of MS effects on the Stroop task. Either (1) MS does not work through a basic cognitive mechanism of spreading activation in a conceptual network, and can thus not be measured with a Stroop task, (2) our Stroop methodology was not suitable to register changes in availability of cognitive constructs, or (3) the standard MS manipulation does not robustly produce cognitive effects (at least not in the form described in the literature). Using a computer mouse for Stroop responses may produce some random variation in response times, but this should be compensated for by the high number of Stroop trials.

To the best of our knowledge, no former experiments have tested a direct effect of MS on processing speed of death-related concepts, or an indirect effect on processing speed of concepts such as social words that are assumed to be causally linked with mortality. Some studies (e.g. Gailliot et al., 2006) have suggested that MS slows down Stroop processing in general, but without using Stroop words with relevant/irrelevant content. This leaves us without an established framework to evaluate whether Stroop is a suitable approach to test the mechanisms assumed to underlie the MS effects. We encourage further testing of this approach in future studies.

\section{No MS interaction effect on psychophysiology}

The TMT assumes that MS leads to an uncomfortable state that motivates the affirmation of one's cultural values (Arndt et al., 2001; Delaney and Brodie, 2000; Henry et al., 2010; Lane et al., 2009; Routledge et al., 2010; Schuler et al., 2017; Silveira et al., 2013). We assumed that such a change of state could be detectable in psychophysiology. However, the manipulation check in Experiment 1 (E1MC2) failed to show a significant effect of decreased HRV in the MS condition. An effect 
of MS on HRV may have been indicated (one-tailed $p=$ .065) but did not meet our preregistered alpha level.

A psychophysiological activation effect may have been obscured by individual variation, measurement noise or analysis choices. It is possible that different HRV analysis approaches (such as different artefact smoothing or different analysis windows) could have indicated effects. However, note that there were no significant effects in an additional testing window (during MS stimulation), nor in the interactions between MS and HRV on any of the outcome measures. Given the high volatility of HRV methods to analytic flexibility, we chose to not explore the data outside of our planned analysis. Note that research on physiological indicators of MS appear to have shown mixed results, also within the TMT literature (Arndt, 1999; Rosenblatt et al., 1989).

\section{Effect of MS to decrease pro-sociality}

Both experiments included our novel money-sharing measure of whether MS increased pro-sociality. A conceptual extension of TMT was that MS should lead to increased generosity to people outside of one's family, as a means to having an impact beyond one's life (building on Jonas et al., 2002; Roberts and Maxfield, 2019; Zaleskiewicz et al., 2015). Experiment 1 showed a non-significant tendency of MS leading to increased money-sharing with friends and charity. However, the more robust test in Experiment 2 showed a significant effect in the opposite direction, of less sharing in the MS group. This is difficult to align with the TMT account, as being less generous with friends and strangers cannot be seen to be a culturally dominant value. One could perhaps formulate an ad hoc explanation for why this measure showed opposite effects in the two studies (e.g., different norm salience, different sample populations), or having opposite predictions from our hypothesis (e.g., wanting to retain resources as proximal defence against death). This illustrates how difficult it can be to derive falsifiable predictions from the TMT (see similar issues raised by Martin and Bos, 2014). In adherence to the hypothethico-deductive approach, we caution against interpreting a non-predicted result as supportive evidence for a given hypothesis. Further, one should keep in mind that the pro-social task is novel and unestablished and was included as an exploratory measure.

\section{Replication of experiment design}

In an attempt to account for the current null-findings, one may point to differences between our experimental design and previous studies. For example, there was no explicit delay task before the target measure in Experiment 1 , which previous research has suggested may be necessary for MS effects to occur (Greenberg et al., 2000). On the other hand, one could argue that the Stroop test and the democratic essays filled this role, as they were performed between the MS manipulation and the patriotic essays measure and are ostensibly unrelated to both mortality and patriotism. Further, Experiment 2 used the most common type of delay task (a 20-item mood measure) and still failed to show a MS effect on national patriotism or ingroup identification in a large US sample. In both experiments the patriotic essays were among the first measures following the manipulation and delay task, to ensure a sensitive test of the primary hypothesis. A review (Burke et al., 2010) has argued that MS effects can be shown across a number of experiment procedures, citing examples that use zero, one, two or three delay tasks. As the TMT claims that MS is a fundamental motivator for human behaviour, it would be surprising if it relies on the exact repetition of minor variations in experimental procedure discussed here. In either case, we would argue that Experiment 2 adheres to the crucial design features in the published literature, in having one delay task immediately preceding the central outcome measures.

Following previous research, our experiments also tested whether the outcome variables showed interactions with cognitive style, political orientation, or the manipulation's effectiveness (although these tests are lower powered than the main effect tests). These tests failed to provide any further support for the MS effects.

Based on the MS literature one may argue that the political orientation of the participants should be taken into consideration. A meta-analysis Burke et al., 2013 found that in some cases MS can lead to a general "conservative shift", whereas other studies have found a polarizing response in which pre-existing political attitudes are amplified regardless of their ideological nature ("worldview defense"). In the current replication, we failed to find empirical support for either of these hypotheses. Specifically, we did not find a significant main effect of death reminders on national patriotism. Although participants with right-leaning political views were more supportive of the national-patriotic essay than left-leaning liberal participants, exposure to the death reminder (MS) did not lead to increased national patriotism in this sub-group either.

\section{Sample size and power}

In terms of sample size, Experiment 1 had 50 participants in each condition, while Experiment 2 had 400 in each condition. The sample sizes reported in a metaanalysis covering more than 400 experiments (Burke et 


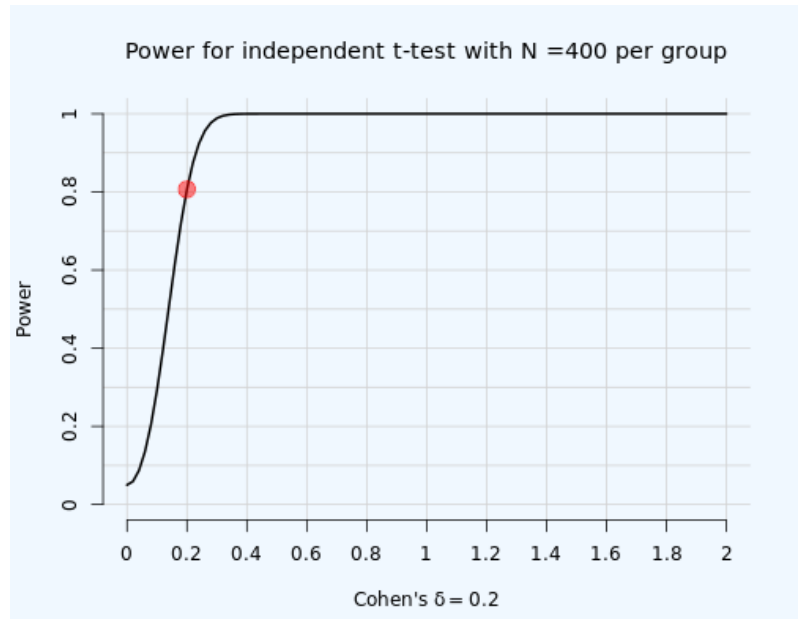

Figure 3. Power-curve for Experiment 2, assuming a quarter of the effect sizes reported in meta-analyses. Adapted from https://shiny.ieis.tue.nl/d_p_power/

al., 2010) can be divided by the first and second predictor variable, to reveal a median sample size per condition to be $n=23.3$. Another meta-analysis of the MS effect on political attitudes (Burke et al., 2013) across 49 experiments reported an average effect size of $\mathrm{d}=$ 1.15 , and a similarly calculated median sample size of $\mathrm{n}=25.9$. Some of the studies may also have had additional, undeclared predictors (argued by John et al., 2012 , to be common in psychology research). We therefore consider Experiment 2 to be a high-powered test of the traditional MS hypothesis. For an overview, see the power-curve in Figure 3 for the statistical power Experiment 2 would have to detect a broad range of effects of different sizes.

Both experiments were thus respectively larger and considerably larger than the studies on MS effects in the literature. These samples are sufficient to detect the effects reported in the literature. However, in replication studies one may be concerned that the originally reported effects are inflated, which should be compensated for in assuming smaller actual effects. Burke and colleagues (2010) reported the average MS effect size to be $\mathrm{d}=0.82$. The statistical power for Experiment 1 had a $99 \%$ power to detect an effect of this size, and $65 \%$ power to detect an effect of half that size. If one assumes that effect sizes has been severely overestimated in the past, one may still consider Experiment 1 to be underpowered. With its larger sample, Experiment 2 had chances approaching $100 \%$ to detect both the reported meta-analytic effect size and half of it, and $80 \%$ chance to detect effect sizes a quarter of that. We therefore consider Experiment 2 to be a high-powered test of the traditional MS hypothesis. For an overview, see the power-curve in Figure 3 for the statistical power Experiment 2 would have to detect a broad range of different effects sizes.

\section{The use of online samples}

To achieve this level of statistical power, Experiment 2 recruited a large online sample using Amazon's Mechanical Turk, while most of the previous literature has been done in-person in physical labs. There have been some recent concerns about reduced data quality when using services like Mechanical Turk (Chmielewski and Kucker, 2019), calling for better data screening. However, closer examination has indicated that such online samples may in fact be more representative for the general population than the student samples typically used in lab experiments (Buhrmester et al., 2011; Buhrmester et al., 2018). Moreover, some studies have indicated that online participants at Mechanical Turk pay closer attention to study instructions than student samples (Hauser and Schwarz, 2016) and provide comparable data quality (Kees et al., 2017). In our view, this suggests that in relatively short and focused survey experiments online samples can be a valuable trade-off to student samples in order to achieve high-powered studies. We recommend that future research develop beyond convenience samples of college students and Mechanical Turk, by testing and replicating focal hypotheses in large-scale representative samples. The validity would be supported if similar results are obtained across different participant samples and methodological approaches.

It could also be argued that the MS phenomenon hinges on the person-to-person interaction that arises in lab-experiments. It should be noted that the literature does not specify that MS is restricted to such conditions or that aspects of the interaction with the experimenter that in itself that causes the effect. If the social interaction is essential for the effect (and it can be shown that transparent and high-powered in-person lab procedures can reliably produce the MS effect), the boundary condition of the need for a social interaction should be implemented in the theoretical account of the phenomenon. We are not aware of any successful replication satisfying these criteria.

A different approach to criticize the use of online samples could be to argue that the MS manipulation may not be attended to or taken seriously when presented online. In response to these concerns, we conducted a manual inspection of the written responses to the traditional MS manipulation task (see Storelv and Sætrevik, 2021, for details). If a notable proportion of our sample did not provide meaningful reflections about the topic they were assigned to write about (death or 
toothache), that would suggest that the overall attention level and corresponding data quality were low. This possibility was rejected since the manipulation check E2MC2 found that $98 \%$ of the online sample provided valid responses to the manipulation. We should note that non-compliance to the manipulation may also occur in in-person studies, and in contrast to our study the traditional MS studies do not typically report manipulation checks.

\section{Failed replication of mortality salience}

A possible explanation for the current null-findings is that the MS effect is less robust than previously assumed. As with the majority of psychological research preceding the recent awareness of fundamental methodological issues (Munafò et al., 2017; Simmons et al., 2011), most MS research has been conducted in a non-transparent way without preregistration or open data. This makes it difficult to assess the extent of unpublished results and undisclosed flexibility in design and analysis in the MS literature.

Independently of our study, a recent "Many Labs 4" project (Klein et al., 2019) has tried to replicate the MS effects across 21 different labs $(\mathrm{N}=2.220)$. These preregistered experiments failed to replicate effects of the original studies, both with and without original author involvement. Note that the initial report from this project was criticised for not adhering closely to the preregistration in determining which studies to include (Chatard et al., 2020). However, a Bayesian multiverse approach to all the Many Labs 4 studies (Haaf et al., 2020) found evidence against MS effects in the majority of the analyses. A recent registered report study also failed to find support for MS across three experiments using established measures (Schindler et al., 2021). These findings mirror the current results, and in addition indicate that methodological expertise in study design is not a likely explanation for the null-findings, nor that the MS effect can be reliably reproduced in lab studies.

The current preregistered null-findings in a controlled lab study and in a large-scale online sample is one of three independent replication projects that have failed to support the MS hypothesis from TMT (Klein et al., 2019; Schindler et al., 2021). This may indicate that the traditional MS effect may not be a robust and replicable phenomenon, despite the high number of past publications (Burke et al., 2013; Burke et al., 2010). At the very least, the current null-findings emphasizes the need for high-powered, preregistered and transparent replication of the traditional MS effect.

\section{Conclusion}

The current study with a Norwegian lab experiment $(\mathrm{N}=101)$ and an American online experiment $(\mathrm{N}=$ 784) aimed to directly replicate an often-reported MS effect on national patriotism, and a previous reported MS effect on ingroup identification. The study further aimed to conceptually replicate the MS effect on support for democratic values, and to explore a potential MS effect on a novel measure of pro-sociality. All these efforts failed to support the predicted MS effects. One of the experiments found a significant MS effect of decreased pro-sociality, but this effect is in the opposite direction of the hypothesis derived from the established literature. The lab experiment was unable to find any effect of MS on processing speed of concepts related to death or social categorization. It may have been indicated that MS led to increased psychophysiological activation, but this failed to reach the cut-off for one-tailed significance in two different analysis windows. We also failed to support interaction effects derived from reasonable interpretations of the MS literature.

Some methodological shortcomings are discussed above. One could claim that while being more transparent and better powered than most of the cited literature, Experiment 1 is nevertheless underpowered and has a rather complex experimental design. However, Experiment 2 can be interpreted alone as a highpowered attempt at directly replicating the central MS effect (Greenberg et al., 1994). Given the claim that MS effects are robust and should generalize across a variety of settings and outcome measures (Burke et al., 2013; Burke et al., 2010; Pyszczynski et al., 2015), it is noteworthy that both our attempts at preregistered replications of the traditional MS effect failed. If one would like to argue that there is solid empirical support for the MS effect in the past literature, one should define the necessary and sufficient conditions to produce the effect (e.g., type and duration of delay task, lab or survey-based data collection, which covariates are necessary), and one should only count studies that fulfil these conditions as having supported the effect.

In our view, the current results show that the basic MS effect is more difficult to reproduce than what is indicated in the literature. It is possible that the MS effect hinges on methodological quirks, specific samples or other boundary conditions that have not been reported or identified in previous research. Variations in theoretical, experimental or analytical approaches may thus have provided different results in the current study. We welcome further research on the proposed MS effect, but we will view the proposed phenomenon with scepticism until such conditions are identified. We actively encourage attempts to replicate the 
current null-findings. Divergent findings could help to identify boundary conditions for the effect (Nosek and Errington, 2020), whereas similar results would further strengthen the current conclusions. Note that the TMT literature has also been claimed to be supported by nonexperiment approaches, such as longitudinal studies following personal events of threat or loss (Ben-Zur and Zeidner, 2009). Although the current study fails to replicate the most commonly cited experimental demonstration of the MS effect, the overall TMT may still be supported by other approaches.

We find it uncontroversial that avoidance of death can be a powerful motivator, and that human psychology is embedded with instincts to favour and support the ingroup. However, it is less obvious that an abstract awareness of mortality could account for a vast array of behaviours not associated with death, or that a subtle death reminder is sufficient to motivate complex behaviour through subconscious processes. Despite our original intention to verify and further explore the nature of MS effects, we found no empirical support for this hypothesis in the present study.

\section{Author Contact}

Bjørn Sætrevik (corresponding author), Department of psychosocial science, Faculty of psychology, University of Bergen, Christies gate 12, 5015 Bergen, Norway. Email: bjorn.satrevik@uib.no ORCID: 0000-00029367-6987. Hallgeir Sjåstad, Norwegian School of Economics. ORCID: 0000-0002-8730-1038.

\section{Conflict of Interest and Funding}

The authors have no financial or non-financial competing interests to report. No benefits impinge on the publication of this article, apart from the expectation that the authors should contribute to objective scientific dissemination as part of their professional role. The interpretation of the results is not biased by the authors' previous professional or scientific work.

Experiment 2 of this research was supported by the Research Council of Norway through its Centres of Excellence Scheme, FAIR project no 262675.

\section{Author Contributions}

Both authors were involved in developing hypotheses and designing both experiments. Author BS conducted Experiment 1, while author HS conducted Experiment 2. Author BS has performed analyses and been in charge of writing the manuscript, while HS has contributed to the process. Author names are ranked to reflect their level of contribution.

\section{Acknowledgements}

We were assisted in the data collection by student researchers Anna Berglann, Ingrid Kleiven, Marte Lovise Aagaard-Nilsen, Silje Johansen, Daniel Gunstveit, Astrid Bergwitz, Isabella Ahlvin, Line Molander, and Frida Ölander.

\section{Open Science Practices}

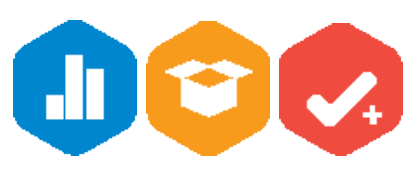

This article earned the Preregistration+, Open Data and the Open Materials badge for preregistering the hypotheses and analyses before data collection, and for making the data and materials openly available. It has been verified that the analysis reproduced the results presented in the article. The entire editorial process, including the open reviews, are published in the online supplement.

\section{References}

Acharya, U., Joseph, K., Kannathal, N., Lim, C., \& Suri, J. (2006). Heart rate variability: A review. Medical and biological engineering and computing, 44(12), 1031-1051.

Arndt, J. (1999). Searching for the terror in terror management: Mortality salience and physiological indices of arousal and affect.

Arndt, J., Allen, J., \& Greenberg, J. (2001). Traces of terror: Subliminal death primes and facial electromyographic indices of affect. Motivation and Emotion, 25(3), 253-277.

Arndt, J., Greenberg, J., \& Cook, A. (2002). Mortality salience and the spreading activation of worldview-relevant constructs: Exploring the cognitive architecture of terror management. Journal of Experimental Psychology: General, $131(3), 307$.

Arndt, J., Greenberg, J., Solomon, S., Pyszczynski, T., \& Simon, L. (1997). Suppression, accessibility of death-related thoughts, and cultural worldview defense: Exploring the psychodynamics of terror management. Journal of personality and social psychology, 73(1), 5.

Becker, E. (1973). The Denial of Death. The Free Press.

Ben-Zur, H., \& Zeidner, M. (2009). Threat to life and risk-taking behaviors: A review of empirical findings and explanatory models. Personality and Social Psychology Review, 13(2), 109-128. 
Buhrmester, M., Kwang, T., \& Gosling, S. (2011). Amazon's Mechanical Turk: A new source of inexpensive, yet high-quality, data? Perspectives on Psychological Science, 6(1), 3-5.

Buhrmester, M., Talaifar, S., \& Gosling, S. (2018). An evaluation of Amazon's Mechanical Turk, its rapid rise, and its effective use. Perspectives on Psychological Science, 13(2), 149-154.

Burke, B., Kosloff, S., \& Landau, M. (2013). Death goes to the polls: A meta-analysis of mortality salience effects on political attitudes. Political Psychology, 34(2), 183-200.

Burke, B., Martens, A., \& Faucher, E. (2010). Two decades of terror management theory: A metaanalysis of mortality salience research. Personality and Social Psychology Review, 14(2), 155195.

Castano, E., Yzerbyt, V., Paladino, M.-P., \& Sacchi, S. (2002). I belong, therefore, I exist: Ingroup identification, ingroup entitativity, and ingroup bias. Personality and Social Psychology Bulletin, 28(2), 135-143.

Chatard, A., Hirschberger, G., \& Pyszczynski, T. (2020). A word of caution about Many Labs 4: If you fail to follow your preregistered plan, you may fail to find a real effect.

Chmielewski, M., \& Kucker, S. (2019). An MTurk Crisis? Shifts in data quality and the impact on study results [Publisher: In]. Social Psychological and Personality Science, 11, 464-473.

Delaney, J., \& Brodie, D. (2000). Effects of short-term psychological stress on the time and frequency domains of heart-rate variability. Perceptual and Motor Skills, 91(2), 515-524.

Doosje, B., Ellemers, N., \& Spears, R. (1995). Perceived intragroup variability as a function of group status and identification. Journal of Experimental Social Psychology, 31(5), 410-436.

Everett, J., Faber, N., \& Crockett, M. (2015). Preferences and beliefs in ingroup favoritism. Frontiers in behavioral neuroscience, 9, 15.

Federico, C., Jost, J., Pierro, A., \& Kruglanski, A. (2007). The need for closure and political attitudes: Final report for the ANES pilot. ANES Pilot Study Report.

Fiedler, K., Kutzner, F., \& Krueger, J. (2012). The long way from -error control to validity proper: Problems with a short-sighted false-positive debate. Perspectives on Psychological Science, 7(6), 661-669.

Gailliot, M., Schmeichel, B., \& Baumeister, R. (2006). Self-regulatory processes defend against the threat of death: Effects of self-control depletion and trait self-control on thoughts and fears of dying. Journal of personality and social psychology, 91(1), 49.

Greenberg, J., Arndt, J., Simon, L., Pyszczynski, T., \& Solomon, S. (2000). Proximal and distal defenses in response to reminders of one's mortality: Evidence of a temporal sequence. Personality and Social Psychology Bulletin, 26(1), 9199.

Greenberg, J., Porteus, J., Simon, L., Pyszczynski, T., \& Solomon, S. (1995). Evidence of a terror management function of cultural icons: The effects of mortality salience on the inappropriate use of cherished cultural symbols. Personality and Social Psychology Bulletin, 21(11), 1221-1228.

Greenberg, J., Pyszczynski, T., \& Solomon, S. (1986). The causes and consequences of a need for selfesteem: A terror management theory. Public self and private self (pp. 189-212). Springer.

Greenberg, J., Pyszczynski, T., Solomon, S., Simon, L., \& Breus, M. (1994). Role of consciousness and accessibility of death-related thoughts in mortality salience effects. Journal of personality and social psychology, 67(4), 627.

Greenberg, J., Simon, L., Pyszczynski, T., Solomon, S., \& Chatel, D. (1992). Terror management and tolerance: Does mortality salience always intensify negative reactions to others who threaten one's worldview? Journal of personality and social psychology, 63(2), 212.

Greenberg, J., Solomon, S., \& Pyszczynski, T. (1997). Terror management theory of self-esteem and cultural worldviews: Empirical assessments and conceptual refinements. Advances in experimental social psychology (pp. 61-139). Elsevier.

Grimson, D., Knowles, S., \& Stahlmann-Brown, P. (2020). How close to home does charity begin? Applied Economics, 52(34), 3700-3708.

Griskevicius, V., Tybur, J., Delton, A., \& Robertson, T. (2011). The influence of mortality and socioeconomic status on risk and delayed rewards: A life history theory approach. Journal of personality and social psychology, 100(6), 1015.

Haaf, J., Hoogeveen, S., Berkhout, S., Gronau, Q., \& Wagenmakers, E.-J. (2020). A Bayesian multiverse analysis of Many Labs 4: Quantifying the evidence against mortality salience.

Hart, J. (2014). Toward an Integrative Theory of Psychological Defense. Perspectives on Psychological Science, 9(1), 19-39. https://doi.org/10.1177/ 1745691613506018

Hauser, D., \& Schwarz, N. (2016). Attentive Turkers: MTurk participants perform better on online 
attention checks than do subject pool participants. Behavior research methods, 48(1), 400407.

Hayes, J., \& Schimel, J. (2018). Unintended effects of measuring implicit processes: The case of death-thought accessibility in mortality salience studies. Journal of Experimental Social Psychology, 74, 257-269.

Hayes, J., Schimel, J., Arndt, J., \& Faucher, E. (2010). A theoretical and empirical review of the deaththought accessibility concept in terror management research. Psychological Bulletin, 136(5), 699.

Hayes, J., Schimel, J., Faucher, E., \& Williams, T. (2008). Evidence for the DTA hypothesis II: Threatening self-esteem increases deaththought accessibility. Journal of Experimental Social Psychology, 44(3), 600-613.

Heine, S., Harihara, M., \& Niiya, Y. (2002). Terror management in Japan. Asian Journal of Social Psychology, 5(3), 187-196.

Henry, E., Bartholow, B., \& Arndt, J. (2010). Death on the brain: Effects of mortality salience on the neural correlates of ingroup and outgroup categorization. Social cognitive and affective neuroscience, 5(1), 77-87.

jamovi project, T. (2019). The jamovi project [Type: (Version 0.9)]. https://www.jamovi.org Retrieved from

John, L., Loewenstein, G., \& Prelec, D. (2012). Measuring the prevalence of questionable research practices with incentives for truth telling. Psychological Science, 0956797611430953.

Jonas, E., Schimel, J., Greenberg, J., \& Pyszczynski, T. (2002). The Scrooge effect: Evidence that mortality salience increases prosocial attitudes and behavior. Personality and Social Psychology Bulletin, 28(10), 1342-1353.

Juhl, J., \& Routledge, C. (2010). Structured terror: Further exploring the effects of mortality salience and personal need for structure on worldview defense. Journal of personality, 78(3), 969-990.

Kahneman, D., Knetsch, J. L., \& Thaler, R. H. (1986). Fairness and the assumptions of economics. Journal of business, S285-S300.

Kees, J., Berry, C., Burton, S., \& Sheehan, K. (2017). An analysis of data quality: Professional panels, student subject pools, and Amazon's Mechanical Turk. Journal of Advertising, 46(1), 141155.

Klein, R., Cook, C., Ebersole, C., Vitiello, C., Nosek, B., Chartier, C., \& Ratliff, K. (2019). Many Labs 4: Failure to Replicate Mortality Salience Ef- fect With and Without Original Author Involvement. https://psyarxiv.com/vef2c

Retrieved from

Lane, R., McRae, K., Reiman, E., Chen, K., Ahern, G., \& Thayer, J. (2009). Neural correlates of heart rate variability during emotion. NeuroImage, 44(1), 213-222.

Martin, L., \& Bos, K. (2014). Beyond terror: Towards a paradigm shift in the study of threat and culture. European Review of Social Psychology, 25(1), 32-70.

Morewedge, C., \& Kahneman, D. (2010). Associative processes in intuitive judgment. Trends in $\mathrm{Cog}$ nitive Sciences, 14(10), 435-440.

Munafò, M., Nosek, B., Bishop, D., Button, K., Chambers, C., Sert, N., \& Ioannidis, J. (2017). A manifesto for reproducible science. Nature Human Behaviour, 1, 0021.

Nosek, B., \& Errington, T. (2020). What is replication? PLoS Biology, 18(3). https : / / doi . org / 10 . 31222/osf.io/u4g6t. What is replication?

Pepper, G., Corby, D., Bamber, R., Smith, H., Wong, N., \& Nettle, D. (2017). The influence of mortality and socioeconomic status on risk and delayed rewards: A replication with British participants. PeerJ, 5, 3580.

Psychology Software Tools, I. (2012). E-Prime 2.0 [Pages: - 2 0]. https://www.pstnet.com Retrieved from

Pyszczynski, T., Solomon, S., \& Greenberg, J. (2015). Thirty years of terror management theory: From genesis to revelation. Advances in experimental social psychology, 52, 1-70.

Roberts, J., \& Maxfield, M. (2019). Mortality Salience and Age Effects on Charitable Donations. American Behavioral Scientist, 0002764219850864.

Rodríguez-Ferreiro, J., Barberia, I., González-Guerra, J., \& Vadillo, M. (2019). Are we truly special and unique? A replication of Goldenberg et al.(2001. Royal Society open science, 6(11), 191114.

Rosenblatt, A., Greenberg, J., Solomon, S., Pyszczynski, T., \& Lyon, D. (1989). Evidence for terror management theory: I. The effects of mortality salience on reactions to those who violate or uphold cultural values. Journal of personality and social psychology, 57(4), 681.

Routledge, C., Ostafin, B., Juhl, J., Sedikides, C., Cathey, C., \& Liao, J. (2010). Adjusting to death: The effects of mortality salience and self-esteem on psychological well-being, growth motivation, 
and maladaptive behavior. Journal of personality and social psychology, 99(6), 897.

Schindler, S., Reinhardt, N., \& Reinhard, M.-A. (2021). Defending one's worldview under mortality salience: Testing the validity of an established idea. Journal of Experimental Social Psychology, 93, 104087.

Schuler, E., Mlynski, C., \& Wright, R. (2017). Influence of mortality salience on effort-related cardiovascular response to an identity-relevant challenge. Motivation Science, 3(2), 164-171.

Silveira, S., Graupmann, V., Agthe, M., Gutyrchik, E., Blautzik, J., Demirçapa, I., \& Reiser, M. (2013). Existential neuroscience: Effects of mortality salience on the neurocognitive processing of attractive opposite-sex faces. Social cognitive and affective neuroscience, 9(10), 1601-1607.

Simmons, J., Nelson, L., \& Simonsohn, U. (2011). Falsepositive psychology: Undisclosed flexibility in data collection and analysis allows presenting anything as significant. Psychological science, 22(11), 1359-1366.

Simon, L., Greenberg, J., Harmon-Jones, E., Solomon, S., Pyszczynski, T., Arndt, J., \& Abend, T. (1997). Terror management and cognitiveexperiential self-theory: Evidence that terror management occurs in the experiential system. Journal of personality and social psychology, $72(5), 1132$.

Singer, P. (2011). The expanding circle: Ethics, evolution, and moral. Princeton University Press.

Sjåstad, H. (2019). Short-sighted greed? focusing on the future promotes reputation-based generosity. Judgment Decision Making, 14(2).
Stets, J. (2006). Identity Theory. In P. Burke (Ed.), Contemporary social psychological theories (pp. 88110). Stanford University Press.

Storelv, S., \& Sætrevik, B. (2021). Nothing is certain except taxes and the other thing: Searching for death anxiety in a large online sample. https: //doi.org/10.31234/osf.io/3tkzq

Trafimow, D., \& Hughes, J. (2012). Testing the death thought suppression and rebound hypothesis: Death thought accessibility following mortality salience decreases during a delay. Social Psychological and Personality Science, 3(5), 622-629.

Turner, J., Oakes, P., Haslam, S., \& McGarty, C. (1994). Self and collective: Cognition and social context. Personality and Social Psychology Bulletin, 20(5), 454-463.

Watson, D., Clark, L., \& Tellegen, A. (1988). Development and validation of brief measures of positive and negative affect: The PANAS scales. Journal of personality and social psychology, 54(6), 1063.

Yen, C.-L., \& Cheng, C.-P. (2013). Researcher effects on mortality salience research: A meta-analytic moderator analysis. Death Studies, 37(7), 636652.

Zaleskiewicz, T., Gasiorowska, A., \& Kesebir, P. (2015). The Scrooge effect revisited: Mortality salience increases the satisfaction derived from prosocial behavior. Journal of Experimental Social Psychology, 59, 67-76. 